\title{
COMMUTATIVITY AND STRUCTURE OF RINGS WITH COMMUTING NILOPENTS
}

\author{
HAZAR ABU-KHUZAM \\ Department of Mathematics \\ University of Petroleum and Minerals \\ Dahran, Saudi Arabia
}

\section{ADIL YAQUB}

Department of Mathematics

University of California

Santa Barbara, California 93106

(Received September 28, 1981)

ABSTRACT. Let $R$ be a ring and let $N$ denote the set of nilpotent elements of $R$. Let $Z$ denote the center of $R$. Suppose that (i) $N$ is commutative, (ii) for every $x$ in $R$ there exists $x^{\prime} \varepsilon<x>$ such that $x-x^{2} x^{\prime} \varepsilon N$, where $\langle x>$ denotes the subring generated by $x$, (iii) for every $x, y$ in $R$, there exists an integer $n=n(x, y) \geq 1$ such that both $(x y)^{n}-(y x)^{n}$ and $(x y)^{n+1}-(y x)^{n+1}$ belong to $z$. Then $R$ is commutative and, in fact, $\mathrm{R}$ is isomorphic to a subdirect sum of nil commutative rings and local commutative rings. It is further shown that both conditions in hypothesis (iii) are essential. The proof uses the structure theory of rings along with some earlier results of the authors.

KEY WOROS AND PHRASES. Nil ring, local ring, subdirect sum, subderectly irreduceble, semisimple ring.

1980 MATHEMATICS SUBJECT CLASSIFICATION CODE. Prumary $16 A 70$.

1. INTRODUCTION.

Recently [1], the authors proved that if $R$ is a semisimple ring with the property that, for all $x, y$ in $R$ there exists an integer $n=n(x, y) \geq 1$ such that $(x y)^{n}-(y x)^{n}$ is in the center of $R$, then $R$ is commutative. This naturally gives rise to the following questinn: what additional conditions are needed to force the commutativity cf 
$R$ when $R$ is an arbitrary ring? In this paper, we establish a theorem which gives some conditions.

\section{MAIN RESULTS.}

In preparation for the proof of the main theorem, we first state the following result which was proved in [2] (also see [3], [4], [5]):

LEMMA 1. Let $R$ be a ring and let $N$ be the set of nilpotent elements of $R$. Suppose that (i) $N$ is commutative, (ii) for every $x$ in $R$ there exists an element $x^{\prime}$ in the subring $\left\langle x>\right.$ generated by $x$ such that $x-x^{2} x^{\prime} \varepsilon N$, (iii) for all a $\varepsilon N$ and $b \varepsilon R$, ba - $a b$ commutes with $b$. Then $R$ is commutative.

With the aid of the above lemma, we are able to prove the following.

MAIN THEOREM. Let $R$ be a ring, $N$ the set of nilpotent elements of $R$, and $Z$ the center of R. Suppose that (i) $N$ is commutative, (ii) for every $x$ in $R$ there exists an element $x^{\prime}$ in the subring $\langle x\rangle$ generated by $x \underline{\text { such that }} x-x^{2} x^{\prime} \varepsilon N$, (iii) for every $x, y$ in $R$, there exists an integer $n=n(x, y) \geq 1$ such that both $(x y)^{n}-(y x)^{n}$ and $(x y)^{n+1}-(y x)^{n+1}$ belong to $z_{c}$ Then $R$ is a subdirect sum of local commutative rings and nil commutative rings.

PROOF. The proof will be broken into several claims.

CLAIM 1. The idempotents of $R$ are all in the center $Z$ of $R$.

For, suppose $e^{2}=e \varepsilon R, x \in R$. By hypothesis (iii), there exists a positive integer $\mathrm{n}$ such that

$$
\{e(e x-e x e+e)\}^{n}-\{(e x-e x e+e) e\}^{n} \varepsilon z .
$$

This reduces to

$$
(e x-e x e+e)-e \varepsilon \cdot z
$$

and hence ex - exe commutes with e. Therefore, ex - exe = e (ex - exe)

$=(e x-e x e) e=0$; that is, ex $=$ exe. Replacing ex - exe $+e$ by $x e-e x e+e$ in the above argument, we obtain xe = exe, and Claim 1 is proved.

CLAIM 2. The set $N$ is a commutative ideal in $R$ and hence $N^{2} \subseteq Z$.

This was essentially proved in [4]. However, for convenience, we re-produce the proof. Let $a \in N, b \in R$ and let $a^{k}=0$.

By hypothesis (ii), 


$$
(a b)^{m}=(a b)^{m+1} c
$$

for some element $c \varepsilon<a b>$ and some positive integer $\mathrm{m}$.

Let

$$
e=(a b)^{m} c^{m} \text {. }
$$

Then, as is readily verified,

$$
(a b)^{m}=(a b)^{m} e, \quad e^{2}=e \varepsilon z \quad(b y \text { Claim } 1) .
$$

Therefore, since e $\varepsilon Z$,

$$
e=e^{2}=e(a b)^{m} c^{m}=a e b(a b)^{m-1} c^{m}=a^{k} e\left\{b(a b)^{m-1} c^{m}\right\}^{k}=0,
$$

since $a^{k}=0$. Thus, $e=0$ and hence by $(2.1),(a b)^{m}=0$. Therefore, ab $\varepsilon$. Similarly, ba $\varepsilon N$. Thus $a b$ and ba are in $N$ for all a $\varepsilon N, b \varepsilon R$. Combining this with hypothesis ( $i$, we conclude that $N$ is a commutative ideal of $R$, and hence $N^{2} \subseteq Z$.

CLAIM 3. If $f: R \rightarrow R^{*}$ is an onto homomorphism, then $f(N)$ coincides with the set of all nilpotent elements of $\mathrm{R} *$.

Again, this was proved in [4], but for convenience we re-produce the proof. Let $d^{*}$ be an arbitrary nilpotent element of $R^{*}$ with $\left(d^{*}\right)^{k}=0$. Choose $d$ in $R$ such that $f(d)=d *$. By hypothesis (ii),

$$
d-d^{2} d^{\prime} \varepsilon N \text { for some } d^{\prime} \varepsilon<d>\text {. }
$$

Observe that

$$
d-d^{k+1}\left(d^{\prime}\right)^{k}=\left(d-d^{2} d^{\prime}\right)+d d^{\prime}\left(d-d^{2} d^{\prime}\right)+\ldots+\left(d d^{\prime}\right)^{k-1}\left(d-d^{2} d^{\prime}\right) .
$$

Since, by Claim 2, $\mathrm{N}$ is an ideal in $\mathrm{R}$, the right side of (2.3) is in $\mathrm{N}($ see (2.2)) and hence

$$
d-d^{k+1}\left(d^{\prime}\right)^{k} \varepsilon N
$$

Recalling that $f(d)=d^{*},\left(d^{*}\right)^{k}=0$, (2.4) now implies that

$$
d^{*}=f(d)=f\left(d-d^{k+1}\left(d^{\prime}\right)^{k}\right) \varepsilon f(N),
$$

and thus $\mathrm{d}^{*} \varepsilon \mathrm{f}(\mathrm{N})$, which proves Claim 3 .

CLAIM 4. Any homomorphic image of $R$ satisfies all the hypotheses (1), (ii), (iii).

This follows at once in view of Claim 3.

To complete the proof of the Main Theorem, first recall that

$$
R \cong a \text { subdirect sum of rings } R_{i}(i \varepsilon \Gamma) \text {; }
$$

each $R_{i}$ is subdirectly irreducible. 
Furthermore, by Claim 4 ,

$$
\text { each } R_{i} \text { satisfies hypotheses (i), (ii), (iii). }
$$

We now distinguish two cases.

Case 1: $R_{i}$ does not have an identity. Let $x \in R_{i}$. By hypothesis (ii), there exists an element $y \varepsilon<x>$ and a positive integer $m$ such that

$$
\mathrm{x}^{\mathrm{m}}=\mathrm{x}^{\mathrm{m}+1} \mathrm{y}, \quad\left(\mathrm{y} \varepsilon<\mathrm{x}>, \mathrm{m} \varepsilon \mathrm{z}^{+}\right) \text {. }
$$

Let

$$
\mathrm{e}=\mathrm{x}^{\mathrm{m}} \mathrm{y}^{\mathrm{m}}, \quad(\mathrm{y} \varepsilon<\mathrm{x}>)
$$

Then, as in the proof of Claim 2 ( see (2.1)),

$$
x^{m}=x^{m} e, \quad e^{2}=e \varepsilon Z_{i} \quad\left(=\text { center of } R_{i} ; \text { see }(2.6) \text { and Claim } 1\right) \text {. }
$$

Since $e$ is a central idempotent in the subdirectly irreducible ring $R_{i}$, and since $R_{i}$ has no identity, $e=0$ and, hence by $(2.8), x^{m}=0$. We have thus shown that $x^{m}=0$ for all $x$ in $R_{i}$; that is, $R_{i}$ is nil.

Hence, $R_{i}$ is a nil commutative ring (see (2.6) and hypothesis (i)).

Case 2: $R_{i}$ has an identity 1 .

Let $x \in R_{i}$. Arguing as in Case 1, the central idempotent element $e$ in (2.7) and (2.8) satisfies

$$
\mathrm{e}=0 \text { or } \mathrm{e}=1
$$

If $\mathrm{e}=0$, then $\mathrm{x}^{\mathrm{m}}=0$ (see (2.8)). On the other hand, if $\mathrm{e}=1$, then by (2.7),

$$
x^{-1}=x^{m-1} y^{m} \varepsilon R_{i} \text {. }
$$

We have thus shown that

$$
R_{i} \text { is a local ring (in Case 2). }
$$

Now, let $u$ be a unit in $R_{i}$ and let $y \in R_{i}$. By (2.6) and hypothesis (iii), there exists an integer $\mathrm{n}=\mathrm{n}(\mathrm{u}, \mathrm{y}) \geq 1$ such that

$$
\left((u y) u^{-1}\right)^{n}-\left(u^{-1}(u y)\right)^{n} \varepsilon z_{i} \quad\left[\text { = center of } R_{i}\right] \text {, }
$$

and hence

$$
\text { uy }{ }^{n}{ }^{-1}-y^{n} \text { commutes with } u \text {. }
$$

Therefore,

$$
\left(u y^{n} u^{-1}-y^{n}\right) u=u\left(u y^{n} u^{-1}-y^{n}\right), \text { and thus }
$$




$$
u y^{n}-y^{n} u=u\left(u y^{n} u^{-1}-y^{n}\right) \text {. }
$$

Multiplying by $u$ on the right side of the above equation, we get

$$
\left(u y^{n}-y^{n} u\right) u=u\left(u y^{n}-y^{n} u\right) ;\left(u=\text { unit in } R_{i}, y \varepsilon R_{i}\right) \text {. }
$$

Setting $y=1+a$, where a is an arbitrary but fixed nilpotent element of $R_{i}$, in (2.11) and recalling that $a^{k} \varepsilon z_{i}$ for all $k \geq 2$ (see Claim 4 and Claim 2), we see that

$$
\begin{gathered}
(u(n a)-(n a) u) u=u(u(n a)-(n a) u) \text {, and thus } \\
n(u a-a u) \text { commutes with } u \text {. }
\end{gathered}
$$

But, by (2.6) and hypothesis (iii), the above argument can be repeated with $n+1$ replacing $n$, to get (see (2.12))

$$
(n+1)(u a-a u) \text { commutes with } u \text {. }
$$

By (2.12) and (2.13), we conclude that

$$
\text { ua - au commutes with } u \text {, ( } u \text { a unit in } R_{i} \text {, a } \varepsilon N_{i} \text { ). }
$$

Moreover, since $\mathrm{N}_{i}$ is commutative (2.14) holds trivially if $\mathrm{u}$ is any nilpotent element of $R_{i}$, and hence by $(2.10)$ and $(2.14)$,

$$
\text { ba - ab commutes with } b \text { for } a l 1 b \varepsilon R_{i} \text {, a } \varepsilon N_{i} \text {. }
$$

Therefore, by Lemma $1, R_{i}$ is commutative, and thus by $(2.10), R_{i}$ is a local commutative ring. This completes the proof of the Main Theorem.

We conclude with the following.

REMARK 1. Our Main Theorem need not be true if we delete one of the two conditions in hypothesis (iii), as a consideration of the following ring shows:

$$
R=\left\{\left(\begin{array}{ccc}
a & b & c \\
0 & a^{2} & 0 \\
0 & 0 & a
\end{array}\right) \quad a, b, c \varepsilon G F(4)\right\}
$$

In this ring, we readily verify that (i) $N^{2}=\{0\}, \quad$ (ii) $x-x^{4} \varepsilon N$ for all $x \varepsilon R$, (iii) $(x y)^{6}=(y x)^{6}$ for all $x, y$ in $R$ (but $(x y)^{7}-(y x)^{7}$ is not in the center of $R$ ). Note that $R$ is not commutative. 
We further remark that we can delete one of the conditions in hypothesis (iii) by fixing $n$ and assuming, in addition, that $R$ is $n$-torsion free. Indeed, the above proof also shows the following.

THEOREM. Let $R$ be a ring and let $n$ be a fixed positive integer such that $(x y)^{n}-$ $(\mathrm{yx})^{\mathrm{n}} \varepsilon \mathrm{Z}$ and $\mathrm{R}$ is $\mathrm{n}$-torsion free. Suppose, further, that the set $\mathrm{N}$ of nilpotents of $R$ is commutative and for every $x$ in $R$, there exists $x^{\prime}$ in $\left\langle x>\right.$ such that $x^{-} x^{2} x^{\prime}$ $\varepsilon N$. Then $R$ is a subdirect sum of local commutative rings and nil commutative rings.

REMARK 2. Our Main Theorem remains valid if we replace the exponent $(n+1)$ in hypothesis (iii) by $\mathrm{m}$, where $\mathrm{m}$ is any positive integer relatively prime to $\mathrm{n}$. Indeed, the only change in the above proof takes place in $(2.13)$, where $(n+1)$ now gets replaced by $\mathrm{m}$, but this does not affect the conclusion in (2.14) or the rest of the proof.

\section{REFERENCES}

1. ABU-KHUZAM, $\mathrm{H}$. and YAQUB, A. A commutativity theorem for division rings, Bul1. Australian Math. Soc., 21 (1980), 43-46.

2. ABU-KHUZAM, H. and YAQUB, A. Some conditions for commutativity of rings with constraints on nilpotent elements, Math. Japonica, 24 (1980), 549-551.

3. HIRANO, Y., IKEHATA, S. and TOMINAGA, H. Commutativitv theorems of Outcalt-Yaqub type, Math. J. Okayama Univ., 21 (1979), 21- 24 .

4. IKEHATA, S. and TOMINAGA, H. A commutativitv theorem, Math. Japonica, 24 (1979), 29-30.

5. PUTCHA. M. and YAQUB, A. Rings with constraints on nilpotent elements and commutators, Studia Scientiarum Mathematicarum Hungarica, 12 (1977), 193-197. 


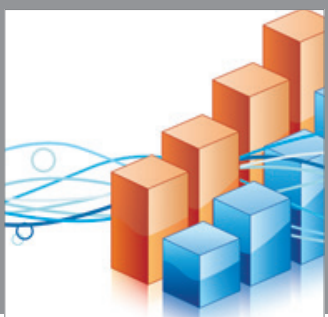

Advances in

Operations Research

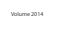

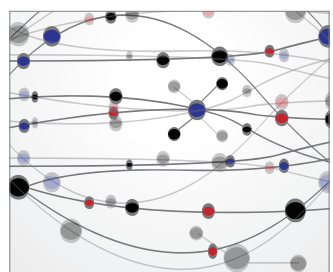

\section{The Scientific} World Journal
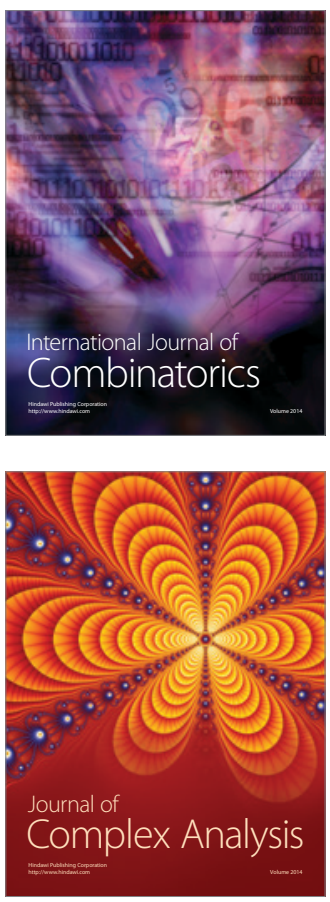

International Journal of

Mathematics and

Mathematical

Sciences
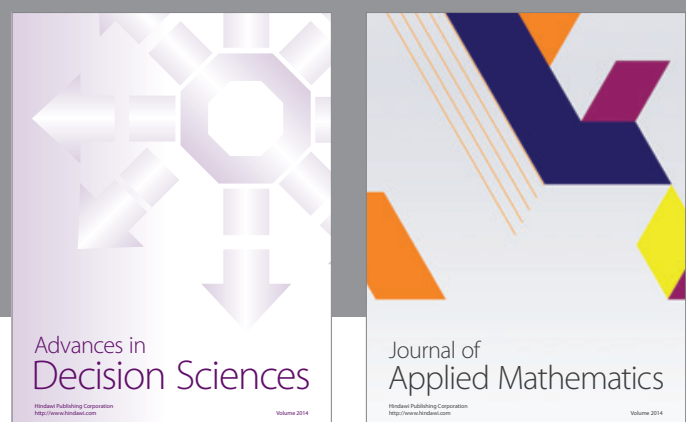

Journal of

Applied Mathematics
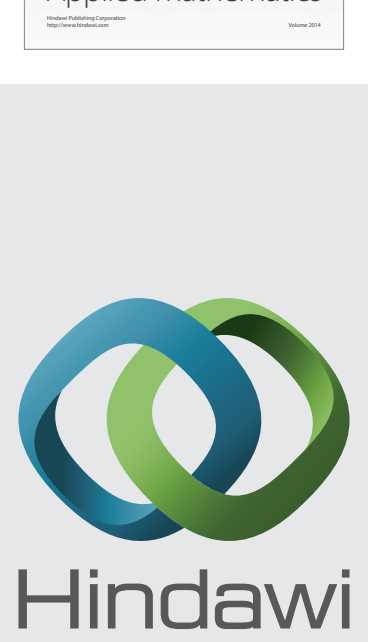

Submit your manuscripts at http://www.hindawi.com
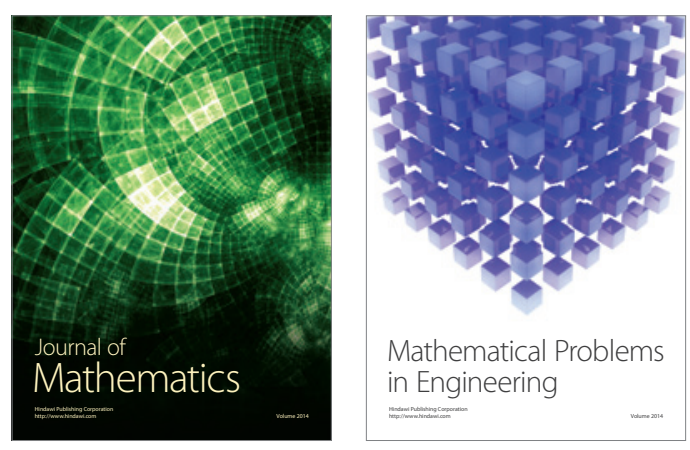

Mathematical Problems in Engineering
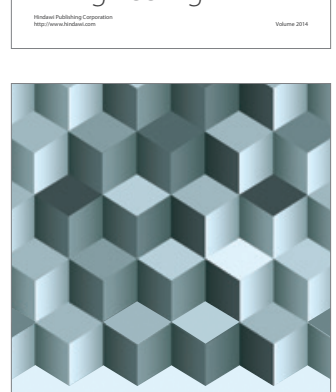

Journal of

Function Spaces
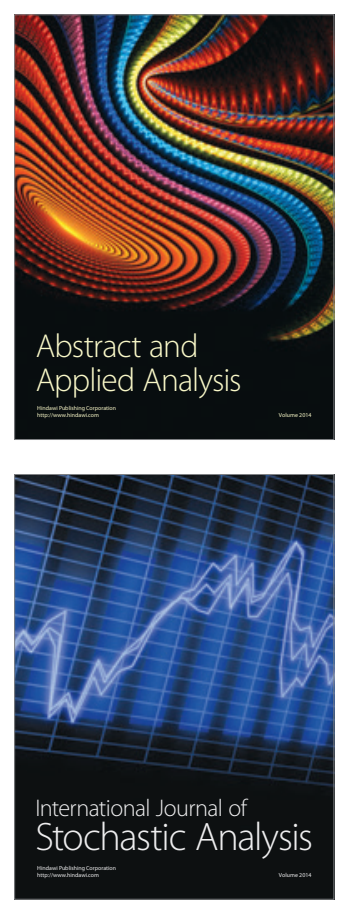

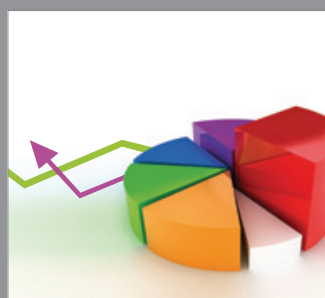

ournal of

Probability and Statistics

Promensencen
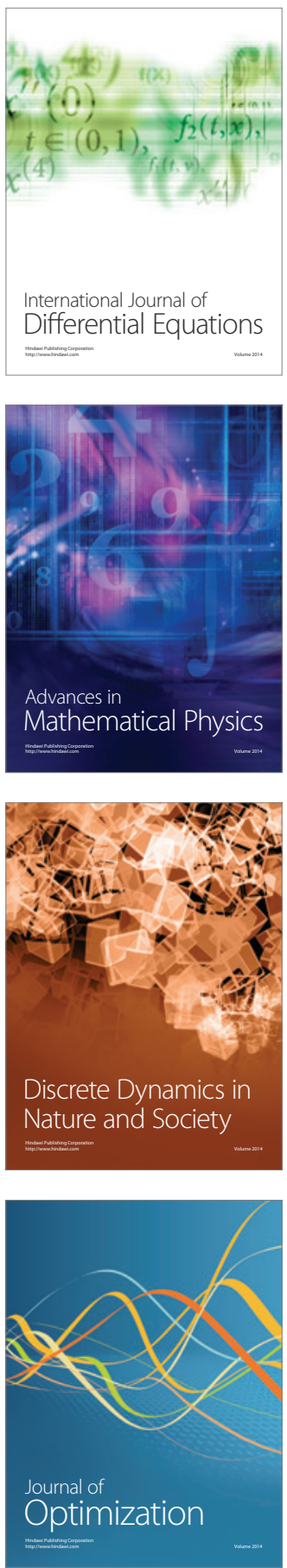